\title{
Evaluation of the relative virulence of novel SARS-CoV-2 variants: a retrospective cohort study in Ontario, Canada
}

\author{
David N. Fisman MD MPH, Ashleigh R. Tuite PhD MPH
}

Cite as: CMAJ 2021 October 25;193:E1619-25. doi: 10.1503/cmaj.211248; early-released October 5, 2021

See related article at www.cmaj.ca/lookup/doi/10.1503/cmaj.211656

\begin{abstract}
Background: Between February and June 2021, the initial wild-type strains of SARS-CoV-2 were supplanted in Ontario, Canada, by new variants of concern (VOCs), first those with the N501Y mutation (i.e., Alpha/B1.1.17, Beta/B.1.351 and Gamma/P.1 variants) and then the Delta/B.1.617 variant. The increased transmissibility of these VOCs has been documented, but knowledge about their virulence is limited. We used Ontario's COVID-19 case data to evaluate the virulence of these VOCs compared with nonVOC SARS-CoV-2 strains, as measured by risk of hospitalization, intensive care unit (ICU) admission and death.
\end{abstract}

Methods: We created a retrospective cohort of people in Ontario who tested positive for SARS-CoV-2 and were screened for VOCs, with dates of test report between Feb. 7 and June 27, 2021. We constructed mixed-effect logistic regression models with hospitalization, ICU admission and death as outcome variables. We adjusted models for age, sex, time, vaccination status, comorbidities and pregnancy status. We included health units as random intercepts.

Results: Our cohort included 212326 people. Compared with non-VOC SARSCoV-2 strains, the adjusted elevation in risk associated with N501Y-positive variants was 52\% (95\% confidence interval [Cl] 42\%-63\%) for hospitalization, $89 \%$ (95\% Cl 67\%-117\%) for ICU admission and $51 \%(95 \% \mathrm{Cl} 30 \%-78 \%)$ for death. Increased risk with the Delta variant was more pronounced at $108 \%(95 \% \mathrm{Cl}$ $78 \%-140 \%$ ) for hospitalization, $235 \%$ (95\% Cl 160\%-331\%) for ICU admission and $133 \%$ (95\% Cl 54\%-231\%) for death.

Interpretation: The increasing virulence of SARS-CoV-2 VOCs will lead to a considerably larger, and more deadly, pandemic than would have occurred in the absence of the emergence of VOCs.
$\mathbf{N}$ ovel SARS-CoV-2 variants of concern (VOCs), including viral lineages carrying the N501Y (Alpha/B.1.1.7) or both the N501Y and E484K mutations (Beta/B.1.351 and Gamma /P.1), were first identified in Ontario, Canada, in December 2020. ${ }^{1}$ Although initially uncommon in Ontario, these VOCs outcompeted earlier SARS-CoV-2 lineages and, as of late April 2021, were responsible for almost all new infections in Ontario, with Alpha the most prevalent lineage. ${ }^{1}$ In April 2021, the B.1.617.2 variant, now known as Delta under the revised nomenclature from the World Health Organization, emerged in the province, outcompeted earlier VOCs and, by July 2021, represented most infections in the province. ${ }^{2,3}$

This serial replacement by emerging variants reflects progressively higher effective reproduction numbers that allow novel variants to outcompete previously dominant strains in the face of identical measures to control spread of infection. ${ }^{4-6}$ However, VOCs are also concerning because emerging evidence points to increased virulence, with increased risk of hospitalization, intensive care unit (ICU) admission and death, after adjustment for age and other predictive factors among patients with $V O C$ infections. ${ }^{7-10}$ Although the increased virulence of strains with the N501Y mutation relative to strains that lack this mutation has been described, ${ }^{7-9}$ only limited information is available on the virulence of infection with the Delta variant, relative to earlier N501Y-positive VOCs (i.e., Alpha, Beta and Gamma)..$^{10-12}$ Our objectives were to evaluate the virulence of N501Y-positive variants relative to earlier SARS-CoV-2 lineages and to evaluate the virulence of the Delta variant of SARS-CoV-2 relative to N501Y-positive VOCs using Ontario's COVID-19 case data.

\section{Methods}

We created a retrospective cohort of people in Ontario who tested positive for SARS-CoV-2 and were screened for VOCs, with dates of test report between Feb. 7 and June 27, 2021. We report the study in accordance with the Strengthening the Reporting of Observational Studies in Epidemiology (STROBE) guidelines for observational research. ${ }^{13}$ 


\section{Data sources and variables}

We extracted case information, including results of screening tests and whole genome sequencing, to identify VOCs from the Ontario Case and Contact Management (CCM) database, as described elsewhere. ${ }^{14}$ The CCM database is derived from the case records of Ontario's public health units and includes information on whether the person infected with SARS-CoV-2 lived in a long-term care institution, had comorbidities, was admitted to hospital or the ICU and whether the person died. We obtained data on vaccination status, including dates of administration of first and second doses of approved SARS-CoV-2 vaccines (where relevant), from COVaxON, a centralized COVID-19 vaccine information system for the province. We linked the COVaxON and the CCM databases using a unique identifier used in both.

The approach for identifying VOCs has changed throughout the pandemic. Although cases of COVID-19 caused by VOCs were identified in Ontario beginning in December 2020, systematic screening for SARS-CoV-2 lineage was not implemented until February 2021, after which time all specimens positive for SARSCoV-2 with a cycle threshold value of 35 or less on testing with the polymerase chain reaction (PCR) were screened for the N501Y mutation. Screening used a single nucleotide polymorphism, real-time PCR assay developed at the Public Health Ontario Laboratory. On Mar. 22, 2021, the province initiated universal screening for N501Y and E484K mutations using a multiplex real-time PCR assay on all specimens testing positive for SARS-CoV-2 with a cycle threshold value of 35 or less. Initially, all specimens with the N501Y or E484K mutation and a cycle threshold value of 30 or less were sequenced; however, as of June 2021, routine whole genome sequencing was no longer performed on specimens without E484K, under the presumption that such specimens were of the Alpha lineage. Whole genome sequencing was performed on a $5 \%$ sample of screened specimens for surveillance purposes, regardless of the presence of mutations; this increased to $10 \%$ and $50 \%$ of screened specimens on May 2 and May 30, 2021, respectively.

By late April 2021, more than 90\% of infections in Ontario were screen-positive for N501Y; subsequently, N501Y mutations became less common, and N501Y-negative specimens subjected to whole genome sequencing were shown to be predominantly Delta variant, with Delta representing more than $60 \%$ of new infections in Ontario on July 1, 2021.,2,3

\section{Study population}

We restricted our analysis to people whose viral isolate had been screened for a VOC. We classified cases as N501Y-positive (screening positive for N501Y or identified as Alpha, Beta or Gamma by whole genome sequencing), probable Delta variant (as identified by whole genome sequencing at any point or screening negative for N501Y and any other mutations from May 1, 2021, onward) or not VOC (all cases negative for N501Y between Feb. 7 and Apr. 30, 2021, and all non-Delta cases testing positive for a mutation other than N501Y, regardless of date). We excluded people for whom VOC screening information was not available or for whom screening could not be completed. We restricted our analysis to people without a record of long-term care residence, given the distinctive epidemiology and severity of SARS-CoV-2 infection in residents of long-term care homes in Ontario. ${ }^{15,16}$

We extracted the data set on July 13, 2021; the most recent test report date was July 12,2021 . We included cases with a test report date at least 14 days prior (June 27, 2021) to account for delays between testing and occurrence of hospitalization, ICU admission and death. We categorized people as fully vaccinated if the case episode date occurred 7 or more days after receipt of the second dose; partially vaccinated if the case episode date occurred 14 days or more after receipt of the first dose but less than 7 days ${ }^{17}$ after receipt of the second dose and unvaccinated, for all other cases. ${ }^{18,19}$

\section{Statistical analysis}

We constructed mixed-effect logistic regression models with hospitalization, ICU admission and death as dependent variables. We adjusted models for age by 10-year increments (as the effect of age on risk of death in people infected with SARS-CoV-2 in Ontario is log-linear), male sex, time (modelled as a week-on-week linear trend), vaccination status and any documented major comorbidity (including asthma, chronic obstructive pulmonary disease, hematological disease, liver disease, cardiac disease, diabetes, immune compromise, renal disease, neurologic disease, malignant disease or obesity). We also adjusted models for hospitalization and ICU for pregnancy, but not models evaluating death because fatalities among pregnant people were rare. Given the geographic variation in prevalence of variants, we included health units as random intercepts. We calculated confidence intervals by parametric bootstrap, assuming fixed effects. As hospitalization, ICU admission and death were relatively rare among cases, we considered odds ratios to approximate relative risks under the "rare disease assumption." ${ }^{20}$ To compare the virulence of Delta variant directly to N501Y-positive VOCs, we also conducted restriction analyses in which we constructed models after exclusion of all non-VOC cases.

We conducted all analyses in R version 4.1.0 using the Ime4 and broom.mixed packages.

\section{Ethics approval}

We received ethics approval for this study from the Research Ethics Board at the University of Toronto (\#00039239).

\section{Results}

We included 212326 cases in our analysis, with SARS-CoV-2 test report dates between Feb. 7 and June 27, 2021 (Figure 1). Figure 2 shows changes over time in the incidence of infections by VOC status and in the proportion of infections that resulted in hospitalization, ICU admission or death. Among all reported cases, $22.4 \%$ were infections with non-VOC, $76.7 \%$ were infections with N501Y-positive VOCs, and $2.8 \%$ were classified as probable Delta infections. In crude comparisons, the number of admissions to hospital and ICU differed significantly between variants (Table 1). People with N501Y-positive variants and probable Delta infection were significantly younger and less likely to have comorbidities 


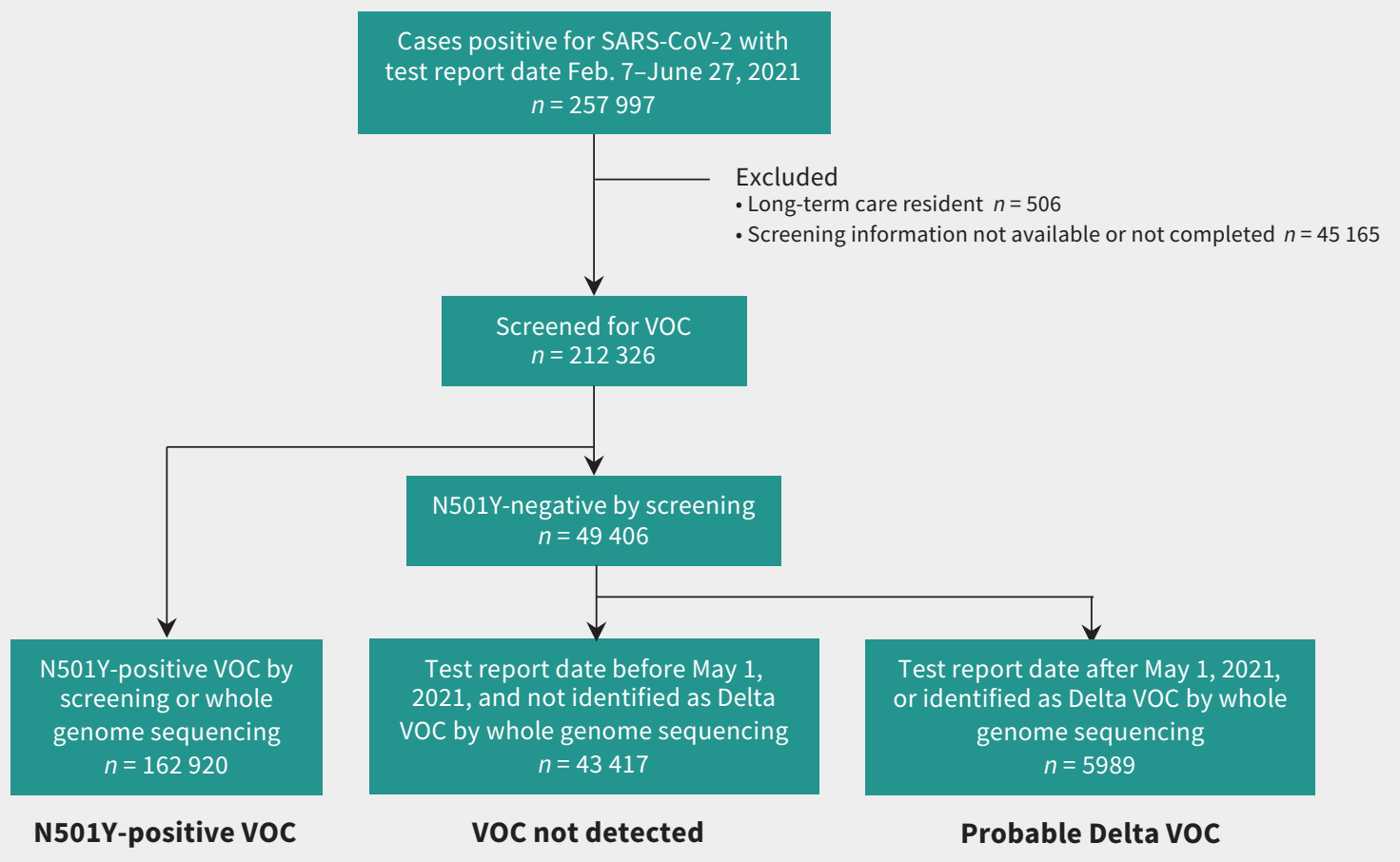

Figure 1: Flow diagram showing inclusion and exclusion of cases from Ontario's Case and Contact Management database in construction of the data set used for this study, as well as the schema used to assign variant of concern (VOC) status.

than those with non-VOC infections. Infections by N501Y-positive variants were significantly more common in the Greater Toronto and Hamilton Area (the province's largest metropolitan area); Delta variant infections were significantly less common in Ottawa (the province's second largest metropolitan area).

After adjustment for age, sex, vaccination status, comorbidities, health unit and temporal trend, we observed large and significant increases in the risk of hospitalization, ICU admission and death with both N501Y-positive VOC and probable Delta infections, relative to non-VOC infections (Table 2). Adjusted risks of hospitalization, ICU admission and death were 52\% (95\% confidence interval $[\mathrm{Cl}]$ 42\%-63\%), 89\% (95\% Cl 67\%-117\%) and 51\% (95\% Cl 30\%-78\%) higher, respectively, with N501Y-positive VOC than with non-VOC infections; respective differences between non-VOC and Delta infections were 108\% (95\% Cl 78\%-140\%), 235\% (95\% Cl 160\%-331\%) and 133\% (95\% Cl 54\%-231\%).

In restriction analyses that excluded non-VOC cases from models, we found a significant increase in risk with Delta infections relative to N501Y-positive VOC infections for hospitalization (adjusted odds ratio [OR] 1.45, 95\% Cl 1.27-1.64), ICU admission (adjusted OR 2.01, 95\% Cl 1.60-2.47) and death (adjusted OR 1.69, 95\% Cl 1.16-2.35).

\section{Interpretation}

We have shown the increased virulence of novel SARS-CoV-2 variants in Ontario, extending the existing evidence base ${ }^{7-9}$ to show the enhanced relative virulence of the Delta variant. Recently published analyses from England and Scotland have shown that
Delta variant infection increases the risk of emergency department use and hospitalization, ${ }^{10,12}$ and a study in Singapore showed an increase in the risk of a composite severity outcome (i.e., oxygen requirement, ICU admission or death) and pneumonia in people with Delta variant infections. ${ }^{11}$ Our study adds to this by showing that Delta variant infection is associated with an increased risk of ICU admission and death, relative to non-VOC and N501Y-positive VOCs.

In our study, VOCs with the N501Y mutation were associated with a markedly increased risk of hospitalization, ICU admission and death among infected individuals in Ontario. The Delta variant, which has now supplanted other VOCs in Ontario, has increased these risks even further. People infected with VOCs were, on average, younger and less likely to have comorbid conditions than those infected with non-VOC, but nonetheless had higher crude risks of hospitalization and ICU admission. Once we adjusted for confounding factors such as age, vaccination status, comorbidity and temporal trends, elevated per-infection risk, including risk of death, remained markedly higher with VOCs, and with the Delta variant in particular. Indeed, given the relatively small number of Delta infections in our study, it is remarkable that we detected a clear and significant elevated risk of uncommon, delayed outcomes, such as death.

The emergence of novel SARS-CoV-2 VOCs has slowed progress against the pandemic in 3 distinct ways, namely by increasing transmissibility and the virus' reproduction number, by increasing immune escape and diminishing vaccine effectiveness ${ }^{21,22}$ and by increasing the virulence of SARS-CoV-2 infection. Canada has now 
A

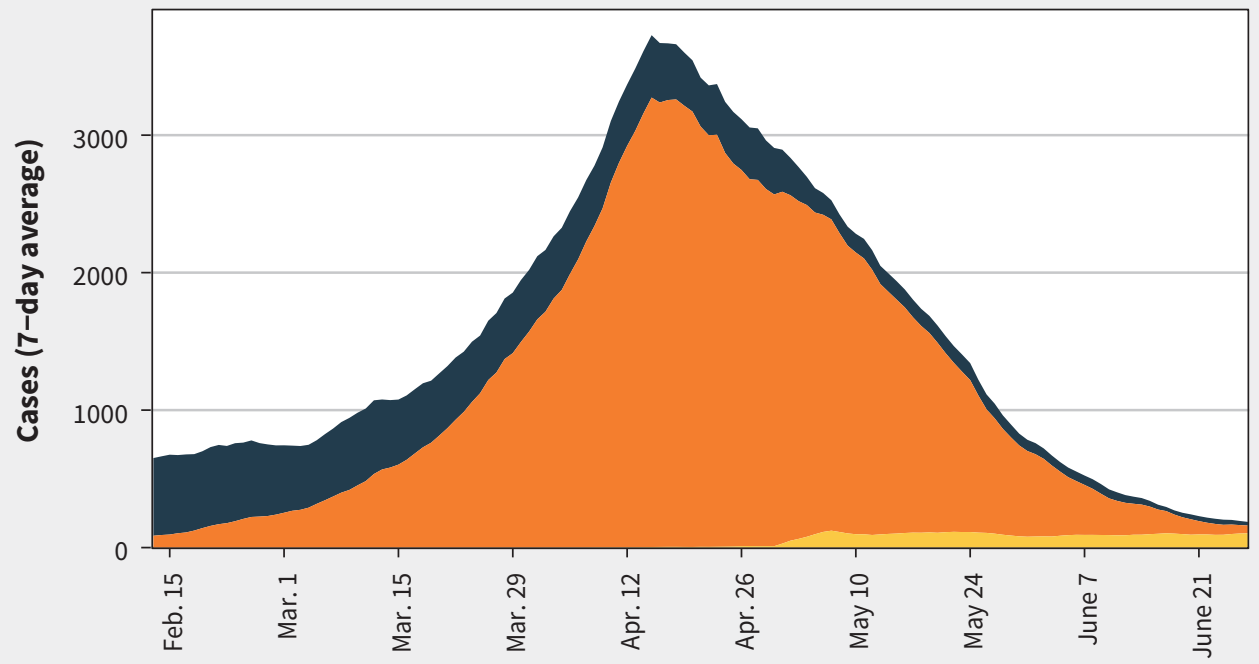

Test report date

\section{Non-VOC \\ N501Y+ VOC \\ Probable Delta VOC}

B

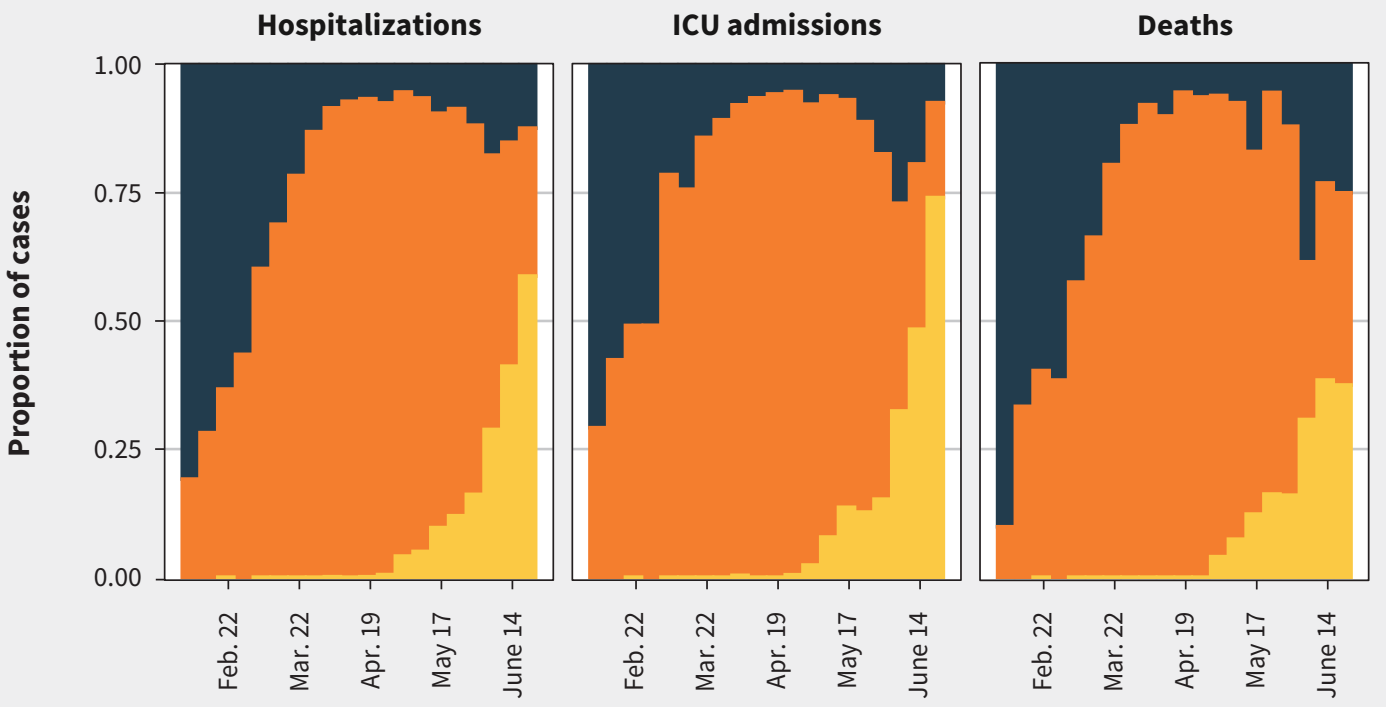

Test report week

Figure 2: Trends in SARS-CoV-2 case occurrence and distribution of hospitalizations, intensive care unit (ICU) admission and deaths by variants of concern (VOC) status in Ontario, Canada. (A) Cases of reported SARS-CoV-2 infection by test report date, presented as the 7-day average for cases reported over the study period. (B) Distribution of cases hospitalized for COVID-19, cases admitted to ICU and deaths by VOC status, by week of test report. Cases are coloured by assigned VOC status. Before May 1, 2021, Delta cases were detected only by whole genome sequencing. After May 1, 2021, all screened specimens not identified as an N501Y-positive VOC (N501Y+ VOC) or another variant were classified as probable Delta VOC infections. 
Table 1: Characteristics of study cohort by variant of concern status

No. $(\%)$ of patients*

\begin{tabular}{|c|c|c|c|c|c|}
\hline Variable & $\begin{array}{c}\text { Total } \\
n=212326\end{array}$ & $\begin{array}{c}\text { VOC not detected } \\
n=43417\end{array}$ & $\begin{array}{l}\text { N501Y-positive VOC } \\
n=162920\end{array}$ & $\begin{array}{c}\text { Probable Delta VOC } \\
\qquad n=5989\end{array}$ & $p$ value \\
\hline Hospitalized & $11038(5.2)$ & $1914(4.4)$ & $8775(5.4)$ & $349(5.8)$ & $<0.001$ \\
\hline Admitted to ICU & $2324(1.1)$ & $337(0.8)$ & $1897(1.2)$ & $90(1.5)$ & $<0.001$ \\
\hline Died & $1820(0.9)$ & $384(0.9)$ & $1396(0.9)$ & $40(0.7)$ & 0.23 \\
\hline Sex, male & $107582(50.7)$ & $22023(50.7)$ & $82505(50.6)$ & 3054 (51.0) & 0.84 \\
\hline Age, yr & & & & & $<0.001$ \\
\hline$<10$ & $15266(7.2)$ & $3016(6.9)$ & $11732(7.2)$ & $518(8.6)$ & \\
\hline $10-19$ & $25390(12.0)$ & $5209(12.0)$ & $19508(12.0)$ & $673(11.2)$ & \\
\hline $20-29$ & $43648(20.6)$ & $8612(19.8)$ & $33640(20.6)$ & $1396(23.3)$ & \\
\hline $30-39$ & 35983 (16.9) & $7117(16.4)$ & $27768(17.0)$ & $1098(18.3)$ & \\
\hline $40-49$ & $31768(15.0)$ & $6198(14.3)$ & $24769(15.2)$ & $801(13.4)$ & \\
\hline $50-59$ & $29972(14.1)$ & $6287(14.5)$ & $22920(14.1)$ & $765(12.8)$ & \\
\hline $60-69$ & $18108(8.5)$ & $3971(9.1)$ & $13664(8.4)$ & $473(7.9)$ & \\
\hline $70-79$ & $8018(3.8)$ & $1892(4.4)$ & $5944(3.6)$ & $182(3.0)$ & \\
\hline$\geq 80$ & $4173(2.0)$ & $1115(2.6)$ & $2975(1.8)$ & $83(1.4)$ & \\
\hline \multicolumn{6}{|l|}{ Vaccination status } \\
\hline Partially vaccinated & 72838 (34.3) & $169327(39.0)$ & $54211(33.3)$ & $1700(28.4)$ & $<0.001$ \\
\hline Fully vaccinated & $945(0.4)$ & $142(0.3)$ & $732(0.4)$ & $71(1.2)$ & $<0.001$ \\
\hline Comorbidity $\ddagger$ & $10805(5.1)$ & $2940(6.8)$ & $7547(4.6)$ & $318(5.3)$ & $<0.001$ \\
\hline Pregnant & $555(0.3)$ & $124(0.3)$ & $410(0.2)$ & $21(0.4)$ & 0.18 \\
\hline \multicolumn{6}{|l|}{ Geography } \\
\hline GTHA§ & $156804(73.9)$ & 27535 (63.4) & $125380(77.0)$ & 3889 (64.9) & $<0.001$ \\
\hline Ottawa & $9325(4.4)$ & $1874(4.3)$ & $7334(4.5)$ & $117(2.0)$ & $<0.001$ \\
\hline
\end{tabular}

Note: GTHA = Greater Toronto and Hamilton Area, ICU = intensive care unit, VOC = variant of concern.

*Unless indicated otherwise.

$\dagger p$ values based on $\chi^{2}$ test.

$\ddagger$ Comorbidities include 1 or more of asthma, chronic obstructive pulmonary disease, hematological disease, liver disease, cardiac disease, diabetes, immune

compromise, renal disease, neurologic disease, malignancy, or obesity.

$\S$ Large conurbation in Central Ontario consisting of 6 health units (Toronto, Peel, Durham, Halton, York and Hamilton).

\section{Table 2: Adjusted odds ratios for hospitalization, intensive care unit admission, and death among reported} COVID-19 cases* $^{*}$

\begin{tabular}{|c|c|c|c|}
\hline Variable & $\begin{array}{c}\text { Hospitalization } \\
\text { OR }(95 \% \mathrm{Cl})\end{array}$ & $\begin{array}{l}\text { ICU admission } \\
\text { OR }(95 \% \mathrm{CI})\end{array}$ & $\begin{array}{c}\text { Death } \\
\text { OR }(95 \% \mathrm{Cl})\end{array}$ \\
\hline \multicolumn{4}{|l|}{ VOC status } \\
\hline Non-VOC & Ref. & Ref. & Ref. \\
\hline Probable Delta variant & $2.08(1.78-2.40)$ & $3.35(2.60-4.31)$ & $2.33(1.54-3.31)$ \\
\hline $\mathrm{N} 501 \mathrm{Y}+$ variant & $1.52(1.42-1.63)$ & $1.89(1.67-2.17)$ & $1.51(1.30-1.78)$ \\
\hline Sex, male & $1.29(1.23-1.36)$ & $1.60(1.48-1.75)$ & $1.67(1.50-1.85)$ \\
\hline Age, yr (per 10-year increase) & $2.07(2.04-2.10)$ & $1.96(1.91-2.01)$ & $3.46(3.32-3.59)$ \\
\hline \multicolumn{4}{|l|}{ Vaccination status } \\
\hline Nonvaccinated & Ref. & Ref. & Ref. \\
\hline Partially vaccinated & $0.42(0.40-0.44)$ & $0.28(0.25-0.30)$ & $0.09(0.07-0.10)$ \\
\hline Fully vaccinated & $0.31(0.23-0.43)$ & $0.15(0.04-0.30)$ & $0.21(0.10-0.35)$ \\
\hline Comorbidity $\dagger$ & $2.76(2.59-2.95)$ & $2.58(2.28-2.90)$ & $2.40(2.08-2.71)$ \\
\hline Pregnant & $6.26(4.56-8.43)$ & $6.85(3.59-10.80)$ & - \\
\hline Series week $\ddagger$ & $0.99(0.99-1.00)$ & $0.98(0.97-0.99)$ & $0.96(0.95-0.98)$ \\
\hline
\end{tabular}


achieved one of the highest SARS-CoV-2 vaccination rates in the world, and vaccination has undoubtedly blunted the impact of these VOCs. We found a marked reduction in risk of severe disease and death among both partially and fully vaccinated people in our study, consistent with the findings of a recently posted preprint. ${ }^{19}$ We performed our analysis in a case-only data set, so the effects reported here represent a substantial degree of protection against death conferred by vaccines (about $80 \%-90 \%$ ), even when they fail to prevent infection. Such direct protective effects may help reduce the health impacts of ongoing SARS-CoV-2 transmission in Ontario, even if herd immunity proves elusive, given the high reproduction numbers of VOCs. ${ }^{5,6}$

Even after adjustment for vaccination status, we observed downward temporal trends in case severity, with a week-onweek reduced risk of hospitalization, ICU admission and death, as shown in Table 2. We suspect this decrease may represent attenuation of illness by vaccination in people who would not have been considered partially or fully vaccinated using our definitions because of inadequate time elapsed since vaccination. This decrease may also represent residual confounding by the changing age distribution of cases over time, which may not have been captured by our broad 10-year age categories, given the early prioritization of older adults for vaccination in Ontario. ${ }^{23}$

\section{Limitations}

An important limitation in our analysis is the possibility of our having misclassified early Delta variant infections as non-VOC owing to the absence of routine screening for characteristic Delta mutations, with likely underestimation of the prevalence of the Delta variant before May 2021. Such misclassification means that our estimates of excess risks for Delta are likely biased toward the null. The CCM and COVaxON databases have not been formally validated for research purposes, but are regularly used by analysts and policy-makers to monitor SARS-CoV-2 epidemiology in Ontario and to plan public health responses to the pandemic.

\section{Conclusion}

In Ontario, despite widespread vaccination and VOC infections occurring more frequently in younger and healthier people, VOCs are associated with a substantial increase in virulence, including increased risk of death. The Delta variant is more virulent than previously dominant N501Y-positive VOCs. Combined with increased transmissibility and immune escape, the increased virulence of VOCs represents a substantial escalation in risk to public health during the SARS-CoV-2 pandemic.

\section{References}

1. Tuite AR, Fisman DN, Odutayo A, et al. Ontario COVID-19 Science Advisory Table. COVID-19 hospitalizations, ICU admissions and deaths associated with the new variants of concern. Science Table COVID-19 Advisory for Ontario; 2021. Available: https://covid19-sciencetable.ca/sciencebrief/covid-19-hospitalizations-icu -admissions-and-deaths-associated-with-the-new-variants-of-concern/ (accessed 2021 July 5).

2. Public Health Ontario. SARS-CoV-2 whole genome sequencing in Ontario, September 7, 2021. Toronto: Queen's Printer for Ontario; 2021. Available: https://www.publichealthontario.ca/-/media/documents/ncov/epi/covid-19-sars -cov2-whole-genome-sequencing-epi-summary.pdf?sc_lang=en (accessed 2021 July 5).
3. Public Health Ontario. Estimating the prevalence and growth of SARSCoV-2 variants in Ontario using mutation profiles. Toronto: Queen's Printer for Ontario; 2021. Available: https://www.publichealthontario.ca/-/media/ documents/ncov/epi/covid-19-prevalence-growth-voc-mutation-epi-summary. pdf?sc_lang=en (accessed 2021 July 5).

4. Davies NG, Abbott S, Barnard RC, et al. Estimated transmissibility and impact of SARS-CoV-2 lineage B.1.1.7 in England. Science 2021;372:eabg3055.

5. Brown KA, Joh E, Buchan SA, et al. Inflection in prevalence of SARS-CoV-2 infections missing the N501Y mutation as a marker of rapid Delta (B.1.617.2) lineage expansion in Ontario, Canada. medRxiv 2021 June 25. doi: 10.1101 /2021.06.22.21259349

6. Brown KA, Gubbay J, Hopkins J, et al. Rapid rise of S-gene target failure and the UK variant B.1.1.7 among COVID-19 isolates in the Greater Toronto Area, Canada. medRxiv 2021 Feb. 12. doi: 10.1101/2021.02.09.21251225.

7. Nyberg T, Twohig KA, Harris RJ, et al. Risk of hospital admission for patients with SARS-CoV-2 variant B.1.1.7: cohort analysis. BMJ 2021;373.

8. Bager P, Wohlfahrt J, Fonager J, et al.; Danish Covid-19 Genome Consortium. Risk of hospitalisation associated with infection with SARS-CoV-2 lineage B.1.1.7 in Denmark: an observational cohort study. Lancet Infect Dis 2021 June 22 [Epub ahead of print]. doi: 10.1016/S1473-3099(21)00290-5.

9. Funk T, Pharris A, Spiteri G, et al.; COVID study groups. Characteristics of SARSCoV-2 variants of concern B.1.1.7, B.1.351 or P.1: data from seven EU/EEA countries, weeks 38/2020 to 10/2021. Euro Surveill 2021;26:2100348.

10. Sheikh A, McMenamin J, Taylor B, et al. Public Health Scotland and the EAVE II Collaborators. SARS-CoV-2 Delta VOC in Scotland: demographics, risk of hospital admission, and vaccine effectiveness. Lancet 2021;397:2461-2.

11. Ong SWX, Chiew CJ, Ang LW, et al. Clinical and virological features of SARS CoV-2 variants of concern: a retrospective cohort study comparing B.1.1.7 (Alpha), B.1.315 (Beta), and B.1.617.2 (Delta). Clin Infect Dis 2021 Aug. 23 [Epub ahead of print]. doi: 10.1093/cid/ciab721.

12. Twohig KA, Nyberg T, Zaidi A, et al.; COVID-19 Genomics UK (COG-UK) consortium. Hospital admission and emergency care attendance risk for SARS-CoV-2 delta (B.1.617.2) compared with alpha (B.1.1.7) variants of concern: a cohort study. Lancet Infect Dis 2021 Aug. 27 [Epub ahead of print]. doi: 10.1016/ S1473-3099(21)00475-8.

13. von Elm E, Altman DG, Egger M, et al.; STROBE Initiative. The Strengthening the Reporting of Observational Studies in Epidemiology (STROBE) statement: guidelines for reporting observational studies. Bull World Health Organ 2007;85 867-72.

14. Fisman DN, Greer AL, Brankston G, et al. COVID-19 case age distribution: cor rection for differential testing by age. Ann Intern Med 2021 Aug. 17 [Epub ahead of print]. doi: 10.7326/M20-7003.

15. Stall NM, Jones A, Brown KA, et al. For-profit long-term care homes and the risk of COVID-19 outbreaks and resident deaths. CMAJ 2020;192:E946-55.

16. Fisman DN, Bogoch I, Lapointe-Shaw L, et al. Risk factors associated with mortality among residents with coronavirus disease 2019 (COVID-19) in long-term care facilities in Ontario, Canada. JAMA Netw Open 2020;3:e2015957.

17. Chung H, He S, Nasreen S, et al.; Canadian Immunization Research Network (CIRN) Provincial Collaborative Network (PCN). Effectiveness of BNT162b2 and mRNA-1273 COVID-19 vaccines against symptomatic SARS-CoV-2 infection and severe COVID-19 outcomes in Ontario, Canada. BMJ 2021;374:n1943.

18. Dagan N, Barda N, Kepten E, et al. BNT162b2 mRNA COVID-19 vaccine in a nationwide mass vaccination setting. N Engl J Med 2021;384:1412-23.

19. Nasreen S, He S, Chung H, et al. Effectiveness of COVID-19 vaccines against variants of concern, Canada. medRxiv 2021 July 3. doi: 10.1101/2021.06.28 .21259420 .

20. Greenland S, Thomas DC. On the need for the rare disease assumption in casecontrol studies. Am J Epidemiol 1982;116:547-53.

21. Wibmer CK, Ayres F, Hermanus T, et al. SARS-CoV-2 501Y.V2 escapes neutralization by South African COVID-19 donor plasma. Nat Med 2021;27:622-5.

22. Lopez Bernal J, Andrews N, Gower C, et al. Effectiveness of COVID-19 vaccines against the B.1.617.2 (Delta) Variant. N Engl J Med 2021;385:585-94.

23. Ontario's COVID-19 vaccination plan. Toronto: Ontario Ministry of Health Available: https://covid-19.ontario.ca/ontarios-covid-19-vaccination-plan (accessed 2021 July 13). 
Competing interests: David Fisman has served on advisory boards related to influenza and SARS-CoV-2 vaccines for Seqirus, Pfizer, AstraZeneca and Sanofi-Pasteur, and has served as a legal expert on issues related to COVID-19 epidemiology for the Elementary Teachers Federation of Ontario and the Registered Nurses Association of Ontario. He was previously a member of the Ontario COVID-19 Modelling Consensus Table. Ashleigh Tuite serves on the Ontario COVID-19 Modelling Consensus Table.

This article has been peer reviewed.

Affiliation: Dalla Lana School of Public Health, University of Toronto, Toronto, Ont.

Contributors: Both authors contributed to the conception and design of the work, and the acquisition, analysis, and interpretation of data. Both authors drafted the manuscript, revised it critically for important intellectual content, gave final approval of the version to be published and agreed to be accountable for all aspects of the work.

Content licence: This is an Open Access article distributed in accordance with the terms of the Creative Commons Attribution (CC BY-NC-ND 4.0) licence, which permits use, distribution and reproduction in any medium, provided that the original publication is properly cited, the use is noncommercial (i.e., research or educational use), and no modifications or adaptations are made. See: https://creativecommons.org/licenses/ by-nc-nd/4.0/

Funding: The research was supported by a grant to David Fisman from the Canadian Institutes for Health Research (2019 COVID-19 rapid researching funding, OV4-170360).

Data sharing: Data used for analysis are the property of the Government of Ontario and are not available from the authors. Interested readers are encouraged to contact the authors regarding technical details of the analysis.

Acknowledgements: The authors wish to thank the staff at Public Health Ontario and Ontario's public health units for collecting, sequencing, analyzing, and providing access to the data used for this analysis.

Accepted: Sept. 16, 2021

Correspondence to: David Fisman, david.fisman@utoronto.ca 